\title{
Higher-Order Duality in Nondifferentiable Minimax Programming with Generalized Type I Functions
}

\author{
I. Ahmad · Z. Husain · S. Sharma
}

Published online: 17 December 2008

(C) Springer Science+Business Media, LLC 2008

\begin{abstract}
A unified higher-order dual for a nondifferentiable minimax programming problem is formulated. Weak, strong and strict converse duality theorems are discussed involving generalized higher-order $(F, \alpha, \rho, d)$-Type I functions.
\end{abstract}

Keywords Nondifferentiable programming · Minimax programming · Higher-order duality

\section{Introduction}

Several authors have shown their interest in developing optimality conditions and duality results for minimax programming problems. Schmitendorf (Ref. [1]) considered the following minimax programming problem:

$$
\begin{aligned}
& \text { (P) } \quad \text { Min } \sup _{y \in Y} f(x, y), \\
& \text { s.t. } \quad g(x) \leq 0, \quad x \in R^{n},
\end{aligned}
$$

where $Y$ is a compact subset of $R^{l}$, the functions $f(\cdot, \cdot): R^{n} \times R^{l} \rightarrow R$, and $g(\cdot)$ : $R^{n} \rightarrow R^{m}$ are in $C^{1}$.

In Ref. [1], Schmitendorf established necessary and sufficient optimality conditions for (P) under convexity. Tanimoto (Ref. [2]) applied these optimality conditions

Communicated by P.M. Pardalos.

The research of second author was supported by the Department of Atomic Energy, Government of India, under the NBHM Post Doctoral Fellowship Program 40/9/2005-R\&D II/2398.

I. Ahmad $(\varangle) \cdot$ Z. Husain $\cdot$ S. Sharma

Department of Mathematics, Aligarh Muslim University, Aligarh 202 002, India

e-mail: izharmaths@hotmail.com 
to define a dual problem and derived duality theorems, which were extended for fractional analogue of (P) by several authors (Refs. [3-15]). Liu (Ref. [16]) discussed the second-order duality theorems for $(\mathrm{P})$ using the concepts of second-order $B$-invex and related functions. A comprehensive view of the minimax problem can be seen in Ref. [17].

In this paper, we consider the following nondifferentiable minimax programming problem:

$$
\begin{aligned}
& \text { (NP) } \quad \text { Min } \sup _{y \in Y} f(x, y)+\left(x^{T} B x\right)^{1 / 2}, \\
& \text { s.t. } \quad g(x) \leq 0, \quad x \in R^{n},
\end{aligned}
$$

where $Y$ is a compact subset of $R^{l}, f(\cdot, \cdot): R^{n} \times R^{l} \rightarrow R, g(\cdot): R^{n} \rightarrow R^{m}$ are continuously differentiable functions at $x \in R^{n}$, and $B$ is an $n \times n$ positive semidefinite symmetric matrix. Recently, Mishra and Rueda (Ref. [18]) discussed duality results for a general Mond-Weir type second-order dual of (NP) involving generalized Type-I functions.

In this paper, we formulate a unified higher-order dual of (NP) and establish weak, strong and strict converse duality theorems under higher-order $(F, \alpha, \rho, d)$-Type I assumptions. More precisely, this paper is an extension of the second order duality results of Mishra and Rueda (Ref. [18]) to a class of higher-order duality, and hence, presents an answer of a question raised therein. Many of the previously published results in this class of optimization appear as special cases of our results.

\section{Notations and Preliminary Results}

Let $R^{n}$ be the $n$-dimensional Euclidean space, $R_{+}^{n}$ be its nonnegative orthant and let $X$ be an open set in $R^{n}$. In what follows $\nabla$ stands for the gradient vector with respect to $x$ throughout the paper.

Let $S=\{x \in X: g(x) \leq 0\}$ denote the set of all feasible solutions of (NP). Any point $x \in S$ is called the feasible point of (NP). Let $J=\{1,2, \ldots, m\}$ be an index set. For each $(x, y) \in S \times Y$, we define

$$
\begin{aligned}
& J(x)=\left\{j \in J: g_{j}(x)=0\right\}, \\
& Y(x)=\left\{y \in Y: f(x, y)+\left(x^{T} B x\right)^{1 / 2}=\sup _{z \in Y} f(x, z)+\left(x^{T} B x\right)^{1 / 2}\right\},
\end{aligned}
$$

and

$$
\begin{aligned}
K=\left\{(s, t, \tilde{y}) \in \mathbb{N} \times R_{+}^{s} \times R^{l s}: 1 \leq s \leq n+1, t=\left(t_{1}, t_{2}, \ldots, t_{s}\right) \in R_{+}^{s}\right. \\
\left.\quad \text { with } \sum_{i=1}^{s} t_{i}=1, \tilde{y}=\left(\bar{y}_{1}, \bar{y}_{2}, \ldots, \bar{y}_{s}\right) \text { with } \bar{y}_{i} \in Y(x), i=1,2, \ldots, s\right\} .
\end{aligned}
$$

Definition 2.1 A functional $F: X \times X \times R^{n} \rightarrow R$ is said to be sublinear in its third argument if, $\forall x, \bar{x} \in X$, 
(i) $F\left(x, \bar{x} ; a_{1}+a_{2}\right) \leq F\left(x, \bar{x} ; a_{1}\right)+F\left(x, \bar{x} ; a_{2}\right), \forall a_{1}, a_{2} \in R^{n}$,

(ii) $F(x, \bar{x} ; \alpha a)=\alpha F(x, \bar{x} ; a), \forall \alpha \in R_{+}, a \in R^{n}$.

By (ii), it is clear that $F(x, \bar{x} ; 0 a)=0$.

Liang, Huang and Pardalos (Refs. [19, 20]) introduced the concept of $(F, \alpha, \rho, d)$ convex function, which was further extended to generalized second-order $(F, \alpha, \rho, d)$ convex functions and generalized second-order $(F, \alpha, \rho, d)$-Type I functions by Ahmad and Husain (Ref. [21]) and Hachimi and Aghezzaf (Ref. [22]), respectively. Here, we rewrite the recently introduced definitions of higher-order $(F, \alpha, \rho, d)$ Type I functions (Ref. [23]) for a minimax programming problem (P) as follows:

Let $F$ be sublinear and let $d(\cdot, \cdot): X \times X \rightarrow R$. Let $\rho=\left(\rho^{1}, \rho^{2}\right)$, where $\rho^{1}=$ $\left(\rho_{1}^{1}, \rho_{2}^{1}, \ldots, \rho_{s}^{1}\right) \in R^{s}$ and $\rho^{2}=\left(\rho_{1}^{2}, \rho_{2}^{2}, \ldots, \rho_{m}^{2}\right) \in R^{m}$, and let $\alpha=\left(\alpha^{1}, \alpha^{2}\right)$, where $\alpha^{1}, \alpha^{2}: X \times X \rightarrow R_{+} \backslash\{0\}$. Let $f(\cdot, \cdot): X \times Y(x) \rightarrow R$ and $g(\cdot): X \rightarrow R^{m}$ be differentiable functions at $\bar{x} \in X$.

Definition 2.2 For each $j \in J,\left(f, g_{j}\right)$ is said to be higher-order $(F, \alpha, \rho, d)$-Type I at $\bar{x} \in X$ with respect to $p \in R^{n}$ if, for all $x \in S$ and $\bar{y}_{i} \in Y(x)$,

$$
\begin{aligned}
& f\left(x, \bar{y}_{i}\right) \geq f\left(\bar{x}, \bar{y}_{i}\right)+h\left(\bar{x}, \bar{y}_{i}, p\right)-p^{T} \nabla_{p} h\left(\bar{x}, \bar{y}_{i}, p\right) \\
& +F\left(x, \bar{x} ; \alpha^{1}(x, \bar{x})\left(\nabla_{p} h\left(\bar{x}, \bar{y}_{i}, p\right)\right)\right)+\rho_{i}^{1} d^{2}(x, \bar{x}), \quad i=1,2, \ldots, s, \\
& -\left[g_{j}(\bar{x})+k_{j}(\bar{x}, p)-p^{T} \nabla_{p} k_{j}(\bar{x}, p)\right] \\
& \geq F\left(x, \bar{x} ; \alpha^{2}(x, \bar{x})\left(\nabla_{p} k_{j}(\bar{x}, p)\right)\right)+\rho_{j}^{2} d^{2}(x, \bar{x}) .
\end{aligned}
$$

Definition 2.3 For each $j \in J,\left(f, g_{j}\right)$ is said to be higher-order $(F, \alpha, \rho, d)$ pseudoquasi-Type I at $\bar{x} \in X$ with respect to $p \in R^{n}$ if, for all $x \in S$ and $\bar{y}_{i} \in Y(x)$,

$$
\begin{aligned}
& f\left(x, \bar{y}_{i}\right)<f\left(\bar{x}, \bar{y}_{i}\right)+h\left(\bar{x}, \bar{y}_{i}, p\right)-p^{T} \nabla_{p} h\left(\bar{x}, \bar{y}_{i}, p\right) \\
& \quad \Rightarrow \quad F\left(x, \bar{x} ; \alpha^{1}(x, \bar{x})\left(\nabla_{p} h\left(\bar{x}, \bar{y}_{i}, p\right)\right)\right)<-\rho_{i}^{1} d^{2}(x, \bar{x}), \quad i=1,2, \ldots, s, \\
& -\left[g_{j}(\bar{x})+k_{j}(\bar{x}, p)-p^{T} \nabla_{p} k_{j}(\bar{x}, p)\right] \leq 0 \\
& \quad \Rightarrow \quad F\left(x, \bar{x} ; \alpha^{2}(x, \bar{x})\left(\nabla_{p} k_{j}(\bar{x}, p)\right)\right) \leq-\rho_{j}^{2} d^{2}(x, \bar{x}) .
\end{aligned}
$$

In the above definition, if

$$
\begin{aligned}
& F\left(x, \bar{x} ; \alpha^{1}(x, \bar{x})\left(\nabla_{p} h\left(\bar{x}, \bar{y}_{i}, p\right)\right)\right) \geq-\rho_{i}^{1} d^{2}(x, \bar{x}) \\
& \quad \Rightarrow \quad f\left(x, \bar{y}_{i}\right)>f\left(\bar{x}, \bar{y}_{i}\right)+h\left(\bar{x}, \bar{y}_{i}, p\right)-p^{T} \nabla_{p} h\left(\bar{x}, \bar{y}_{i}, p\right), \quad i=1,2, \ldots, s,
\end{aligned}
$$

then we say that $\left(f, g_{j}\right)$ is higher-order $(F, \alpha, \rho, d)$-strictlypseudoquasi Type I at $\bar{x}$.

Remark 2.1 If $\alpha^{1}(x, \bar{x})=\alpha^{2}(x, \bar{x})=1, h\left(\bar{x}, \bar{y}_{i}, p\right)=p^{T} \nabla f\left(\bar{x}, \bar{y}_{i}\right)+\frac{1}{2} p^{T} \nabla^{2} f\left(\bar{x}, \bar{y}_{i}\right) p$, $\rho_{i}^{1}=0, i=1,2, \ldots, s, k_{j}(\bar{x}, p)=p^{T} \nabla g_{j}(\bar{x})+\frac{1}{2} p^{T} \nabla^{2} g_{j}(\bar{x}) p, \rho_{j}^{2}=0, j \in J$ and $F(x, \bar{x} ; a)=\eta^{T}(x, \bar{x}) a$, where $\eta: S \times X \rightarrow R^{n}$, then the above definitions reduce to that of second-order Type I/second-order pseudoquasi Type I functions given by Mishra and Rueda (Ref. [18]). 
Lemma 2.1 (Generalized Schwartz Inequality) Let B be a positive semidefinite symmetric matrix of order $n$. Then, for all $x, u \in R^{n}$,

$$
x^{T} B u \leq\left(x^{T} B x\right)^{1 / 2}\left(u^{T} B u\right)^{1 / 2} .
$$

We observe that equality holds if $B x=\lambda B u$, for some $\lambda \geq 0$. Evidently, if $u^{T} B u \leq 1$, we have

$$
x^{T} B u \leq\left(x^{T} B x\right)^{1 / 2} .
$$

The following theorem is a particular case of Theorem 3.1 in Ref. [6] and will be needed in the present analysis.

Theorem 2.1 (Necessary Conditions) If $x^{*}$ is a (local or global) solution of (NP) satisfying $x^{* T} B x^{*}>0$, and if $\nabla g_{j}\left(x^{*}\right), j \in J\left(x^{*}\right)$ are linearly independent, then there exist $\left(s^{*}, t^{*}, \tilde{y}^{*}\right) \in K, u^{*} \in R^{n}$, and $\mu^{*} \in R_{+}^{m}$ such that

$$
\begin{aligned}
& \sum_{i=1}^{s^{*}} t_{i}^{*} \nabla f\left(x^{*}, \bar{y}_{i}^{*}\right)+B u^{*}+\sum_{j=1}^{m} \nabla \mu_{j}^{*} g_{j}\left(x^{*}\right)=0, \\
& \sum_{j=1}^{m} \mu_{j}^{*} g_{j}\left(x^{*}\right)=0, \\
& t_{i}^{*} \geq 0, \quad i=1,2, \ldots, s^{*}, \sum_{i=1}^{s^{*}} t_{i}^{*}=1, \\
& u^{* T} B u^{*} \leq 1, \quad\left(x^{* T} B x^{*}\right)^{1 / 2}=x^{* T} B u^{*} .
\end{aligned}
$$

\section{Unified Higher-Order Duality}

In this section, we formulate the following unified higher-order dual of (NP) and derive duality results:

$$
\begin{gathered}
\text { (GD) } \max _{(s, t, \tilde{y}) \in K} \sup _{(z, u, \mu, p) \in H(s, t, \tilde{y})} \sum_{i=1}^{s} t_{i}\left\{f\left(z, \bar{y}_{i}\right)+h\left(z, \bar{y}_{i}, p\right)-p^{T} \nabla_{p} h\left(z, \bar{y}_{i}, p\right)\right\} \\
+z^{T} B u+\sum_{j \in J_{\circ}}\left\{\mu_{j} g_{j}(z)+\mu_{j} k_{j}(z, p)-p^{T} \nabla_{p}\left(\mu_{j} k_{j}(z, p)\right)\right\},
\end{gathered}
$$

where $H(s, t, \tilde{y})$ denotes the set of all $(z, u, \mu, p) \in R^{n} \times R^{n} \times R_{+}^{m} \times R^{n}$ satisfying

$$
\begin{aligned}
& \sum_{i=1}^{s} t_{i} \nabla_{p} h\left(z, \bar{y}_{i}, p\right)+B u+\sum_{j=1}^{m} \nabla_{p}\left(\mu_{j} k_{j}(z, p)\right)=0, \\
& \sum_{j \in J_{\beta}}\left\{\mu_{j} g_{j}(z)+\mu_{j} k_{j}(z, p)-p^{T} \nabla_{p}\left(\mu_{j} k_{j}(z, p)\right)\right\} \geq 0, \quad \beta=1,2, \ldots, r, \\
& u^{T} B u \leq 1,
\end{aligned}
$$


where $J_{\beta} \subseteq J=\{1,2, \ldots, m\}, \beta=0,1,2, \ldots, r$, with $J_{\beta} \cap J_{\gamma}=\emptyset$ if $\beta \neq \gamma$, $\bigcup_{\beta=0}^{r} J_{\beta}=J$. If, for a triplet $(s, t, \tilde{y}) \in K$, the set $H(s, t, \tilde{y})=\emptyset$, then we define the supremum over it to be $-\infty$.

Remark 3.1 Let $h\left(z, \bar{y}_{i}, p\right)=p^{T} \nabla f\left(z, \bar{y}_{i}\right)+\frac{1}{2} p^{T} \nabla^{2} f\left(z, \bar{y}_{i}\right) p, i=1,2, \ldots, s$, and $k_{j}(z, p)=p^{T} \nabla g_{j}(z)+\frac{1}{2} p^{T} \nabla^{2} g_{j}(z) p, j \in J$. Then (GD) reduces to the secondorder dual (Refs. [18, 24]). If, in addition, $B=0$, then we get the dual formulated by Liu (Ref. [16]).

Theorem 3.1 (Weak Duality) Let $x$ and $(z, u, \mu, s, t, \tilde{y}, p)$ be the feasible solutions of $(N P)$ and $(G D)$, respectively. Suppose that

$$
\left[\sum_{i=1}^{s} t_{i} f\left(\cdot, \bar{y}_{i}\right)+(\cdot)^{T} B u+\sum_{j \in J_{\circ}} \mu_{j} g_{j}(\cdot), \sum_{j \in J_{\beta}} \mu_{j} g_{j}(\cdot), \beta=1,2, \ldots, r\right]
$$

is higher-order $(F, \alpha, \rho, d)$-pseudoquasi Type I at $z$ and

$$
\left(\frac{\rho_{1}^{1}}{\alpha^{1}(x, z)}+\frac{\sum_{\beta=1}^{r} \rho_{\beta}^{2}}{\alpha^{2}(x, z)}\right) \geq 0 .
$$

Then

$$
\begin{aligned}
& \sup _{y \in Y} f(x, y)+\left(x^{T} B x\right)^{1 / 2} \\
& \geq \sum_{i=1}^{s} t_{i}\left\{f\left(z, \bar{y}_{i}\right)+h\left(z, \bar{y}_{i}, p\right)-p^{T} \nabla_{p} h\left(z, \bar{y}_{i}, p\right)\right\}+z^{T} B u \\
& \quad+\sum_{j \in J_{\circ}}\left\{\mu_{j} g_{j}(z)+\mu_{j} k_{j}(z, p)-p^{T} \nabla_{p}\left(\mu_{j} k_{j}(z, p)\right)\right\} .
\end{aligned}
$$

Proof Suppose, contrary to the result, that

$$
\begin{aligned}
& \sup _{y \in Y} f(x, y)+\left(x^{T} B x\right)^{1 / 2} \\
& <\sum_{i=1}^{s} t_{i}\left\{f\left(z, \bar{y}_{i}\right)+h\left(z, \bar{y}_{i}, p\right)-p^{T} \nabla_{p} h\left(z, \bar{y}_{i}, p\right)\right\}+z^{T} B u \\
& \quad+\sum_{j \in J_{\circ}}\left\{\mu_{j} g_{j}(z)+\mu_{j} k_{j}(z, p)-p^{T} \nabla_{p}\left(\mu_{j} k_{j}(z, p)\right)\right\} .
\end{aligned}
$$

Thus, we obtain

$$
\begin{aligned}
& f\left(x, \bar{y}_{i}\right)+\left(x^{T} B x\right)^{1 / 2} \\
& \quad<\sum_{i=1}^{s} t_{i}\left\{f\left(z, \bar{y}_{i}\right)+h\left(z, \bar{y}_{i}, p\right)-p^{T} \nabla_{p} h\left(z, \bar{y}_{i}, p\right)\right\}+z^{T} B u
\end{aligned}
$$




$$
+\sum_{j \in J_{\circ}}\left\{\mu_{j} g_{j}(z)+\mu_{j} k_{j}(z, p)-p^{T} \nabla_{p}\left(\mu_{j} k_{j}(z, p)\right)\right\},
$$

for all $\bar{y}_{i} \in Y(x), i=1,2, \ldots, s$.

It follows from $t_{i} \geq 0, i=1,2, \ldots, s$, that

$$
\begin{aligned}
& t_{i}\left[\left(f\left(x, \bar{y}_{i}\right)+\left(x^{T} B x\right)^{1 / 2}\right)-\left(\sum_{i=1}^{s} t_{i}\left\{f\left(z, \bar{y}_{i}\right)+h\left(z, \bar{y}_{i}, p\right)-p^{T} \nabla_{p} h\left(z, \bar{y}_{i}, p\right)\right\}\right.\right. \\
& \left.\left.\quad+z^{T} B u+\sum_{j \in J_{\circ}}\left\{\mu_{j} g_{j}(z)+\mu_{j} k_{j}(z, p)-p^{T} \nabla_{p}\left(\mu_{j} k_{j}(z, p)\right)\right\}\right)\right] \leq 0, \\
& \quad i=1,2, \ldots, s
\end{aligned}
$$

with at least one strict inequality, since $t=\left(t_{1}, t_{2}, \ldots, t_{s}\right) \neq 0$. Taking summation over $i$ and using $\sum_{i=1}^{s} t_{i}=1$, we have

$$
\begin{aligned}
& \sum_{i=1}^{s} t_{i} f\left(x, \bar{y}_{i}\right)+\left(x^{T} B x\right)^{1 / 2} \\
& <\sum_{i=1}^{s} t_{i}\left\{f\left(z, \bar{y}_{i}\right)+h\left(z, \bar{y}_{i}, p\right)-p^{T} \nabla_{p} h\left(z, \bar{y}_{i}, p\right)\right\}+z^{T} B u \\
& \quad+\sum_{j \in J_{\circ}}\left\{\mu_{j} g_{j}(z)+\mu_{j} k_{j}(z, p)-p^{T} \nabla_{p}\left(\mu_{j} k_{j}(z, p)\right)\right\},
\end{aligned}
$$

which, by the feasibility of $x$ for (NP) and $\mu \in R_{+}^{m}$, gives

$$
\begin{aligned}
& \sum_{i=1}^{s} t_{i} f\left(x, \bar{y}_{i}\right)+\left(x^{T} B x\right)^{1 / 2}+\sum_{j \in J_{\circ}} \mu_{j} g_{j}(x) \\
& \quad<\sum_{i=1}^{s} t_{i}\left\{f\left(z, \bar{y}_{i}\right)+h\left(z, \bar{y}_{i}, p\right)-p^{T} \nabla_{p} h\left(z, \bar{y}_{i}, p\right)\right\} \\
& \quad+z^{T} B u+\sum_{j \in J_{\circ}}\left\{\mu_{j} g_{j}(z)+\mu_{j} k_{j}(z, p)-p^{T} \nabla_{p}\left(\mu_{j} k_{j}(z, p)\right)\right\} .
\end{aligned}
$$

It follows from Lemma 2.1 and (3) that

$$
\begin{aligned}
& \sum_{i=1}^{s} t_{i} f\left(x, \bar{y}_{i}\right)+x^{T} B u+\sum_{j \in J_{\circ}} \mu_{j} g_{j}(x) \\
& <\sum_{i=1}^{s} t_{i}\left\{f\left(z, \bar{y}_{i}\right)+h\left(z, \bar{y}_{i}, p\right)-p^{T} \nabla_{p} h\left(z, \bar{y}_{i}, p\right)\right\}+z^{T} B u \\
& \quad+\sum_{j \in J_{\circ}}\left\{\mu_{j} g_{j}(z)+\mu_{j} k_{j}(z, p)-p^{T} \nabla_{p}\left(\mu_{j} k_{j}(z, p)\right)\right\} .
\end{aligned}
$$


Also, from (2), we have

$$
-\sum_{j \in J_{\beta}}\left\{\mu_{j} g_{j}(z)+\mu_{j} k_{j}(z, p)-p^{T} \nabla_{p}\left(\mu_{j} k_{j}(z, p)\right)\right\} \leq 0, \quad \beta=1,2, \ldots, r .
$$

The higher-order $(F, \alpha, \rho, d)$-pseudoquasi Type I assumption on

$$
\left[\sum_{i=1}^{s} t_{i} f\left(\cdot, \bar{y}_{i}\right)+(\cdot)^{T} B u+\sum_{j \in J_{\circ}} \mu_{j} g_{j}(\cdot), \sum_{j \in J_{\beta}} \mu_{j} g_{j}(\cdot), \quad \beta=1,2, \ldots, r\right]
$$

at $z$, with (4) and (5), implies

$$
\begin{aligned}
& F\left(x, z ; \alpha^{1}(x, z)\left\{\sum_{i=1}^{s} t_{i} \nabla_{p} h\left(z, \bar{y}_{i}, p\right)+B u+\sum_{j \in J_{\circ}} \nabla_{p}\left(\mu_{j} k_{j}(z, p)\right)\right\}\right) \\
& \quad<-\rho_{1}^{1} d^{2}(x, z), \\
& F\left(x, z ; \alpha^{2}(x, z) \sum_{j \in J_{\beta}} \nabla_{p}\left(\mu_{j} k_{j}(z, p)\right)\right) \leq-\rho_{\beta}^{2} d^{2}(x, z), \quad \beta=1,2, \ldots, r .
\end{aligned}
$$

By using $\alpha^{1}(x, z)>0, \alpha^{2}(x, z)>0$, and the sublinearity of $F$ in the above inequalities, we summarize to get

$$
\begin{aligned}
& F\left(x, z ; \sum_{i=1}^{s} t_{i} \nabla_{p} h\left(z, \bar{y}_{i}, p\right)+B u+\sum_{j=1}^{m} \nabla_{p}\left(\mu_{j} k_{j}(z, p)\right)\right) \\
& \quad<-\left(\frac{\rho_{1}^{1}}{\alpha^{1}(x, z)}+\frac{\sum_{\beta=1}^{r} \rho_{\beta}^{2}}{\alpha^{2}(x, z)}\right) d^{2}(x, z) .
\end{aligned}
$$

Since

$$
\left(\frac{\rho_{1}^{1}}{\alpha^{1}(x, z)}+\frac{\sum_{\beta=1}^{r} \rho_{\beta}^{2}}{\alpha^{2}(x, z)}\right) \geq 0
$$

inequality (6) yields

$$
F\left(x, z ; \sum_{i=1}^{s} t_{i} \nabla_{p} h\left(z, \bar{y}_{i}, p\right)+B u+\sum_{j=1}^{m} \nabla_{p}\left(\mu_{j} k_{j}(z, p)\right)\right)<0,
$$

which contradicts $(1)$, as $F(x, z ; 0)=0$.

Theorem 3.2 (Strong Duality) Let $x^{*}$ be an optimal solution of (NP) and let $\nabla g_{j}\left(x^{*}\right), j \in J\left(x^{*}\right)$ be linearly independent. Assume that

$$
\begin{aligned}
& h\left(x^{*}, \bar{y}_{i}^{*}, 0\right)=0, \quad \nabla_{p} h\left(x^{*}, \bar{y}_{i}^{*}, 0\right)=\nabla f\left(x^{*}, \bar{y}_{i}^{*}\right), \quad i=1,2, \ldots, s, \\
& k_{j}\left(x^{*}, 0\right)=0, \quad \nabla_{p} k_{j}\left(x^{*}, 0\right)=\nabla g_{j}\left(x^{*}\right), \quad j \in J .
\end{aligned}
$$


Then there exist $\left(s^{*}, t^{*}, \tilde{y}^{*}\right) \in K$ and $\left(x^{*}, u^{*}, \mu^{*}, p^{*}\right) \in H\left(s^{*}, t^{*}, \tilde{y}^{*}\right)$ such that $\left(x^{*}, u^{*}, \mu^{*}, s^{*}, t^{*}, \tilde{y}^{*}, p^{*}=0\right)$ is a feasible solution of (GD) and the two objectives have the same values. Furthermore, if the assumptions of weak duality (Theorem 3.1) hold for all feasible solutions of (NP) and (GD), then $\left(x^{*}, u^{*}, \mu^{*}, s^{*}, t^{*}, \tilde{y}^{*}, p^{*}=0\right)$ is an optimal solution of (GD).

Proof Since $x^{*}$ is an optimal solution of (NP) and $\nabla g_{j}\left(x^{*}\right), j \in J\left(x^{*}\right)$ are linearly independent, by Theorem 2.1, there exist $\left(s^{*}, t^{*}, \tilde{y}^{*}\right) \in K$ and $\left(x^{*}, u^{*}, \mu^{*}, p^{*}\right) \in$ $H\left(s^{*}, t^{*}, \tilde{y}^{*}\right)$ such that

$$
\begin{aligned}
& \sum_{i=1}^{s^{*}} t_{i}^{*} \nabla f\left(x^{*}, \bar{y}_{i}^{*}\right)+B u^{*}+\sum_{j=1}^{m} \nabla \mu_{j}^{*} g_{j}\left(x^{*}\right)=0, \\
& \sum_{j=1}^{m} \mu_{j}^{*} g_{j}\left(x^{*}\right)=0, \\
& t_{i}^{*} \geq 0, \quad i=1,2, \ldots, s^{*}, \quad \sum_{i=1}^{s^{*}} t_{i}^{*}=1, \\
& u^{* T} B u^{*} \leq 1, \\
& \left(x^{* T} B x^{*}\right)^{1 / 2}=x^{* T} B u^{*} .
\end{aligned}
$$

Thus the relations (8)-(11) along with (7) imply that $\left(x^{*}, u^{*}, \mu^{*}, s^{*}, t^{*}, \tilde{y}^{*}, p^{*}=0\right)$ is a feasible solution of (GD). Also (7), (9) and (12) with $p^{*}=0$ show the equality of objective values. Optimality of $\left(x^{*}, u^{*}, \mu^{*}, s^{*}, t^{*}, \tilde{y}^{*}, p^{*}=0\right)$ thus follows from weak duality (Theorem 3.1).

Theorem 3.3 (Strict Converse Duality) Let $x^{*}$ and $\left(z^{*}, u^{*}, \mu^{*}, s^{*}, t^{*}, \tilde{y}^{*}, p^{*}\right)$ be the optimal solutions of (NP) and (GD), respectively. Suppose that

$$
\left[\sum_{i=1}^{s^{*}} t_{i}^{*} f\left(\cdot, \bar{y}_{i}^{*}\right)+(\cdot)^{T} B u^{*}+\sum_{j \in J_{\circ}} \mu_{j}^{*} g_{j}(\cdot), \sum_{j \in J_{\beta}} \mu_{j}^{*} g_{j}(\cdot), \beta=1,2, \ldots, r\right]
$$

is higher-order $(F, \alpha, \rho, d)$-strictly pseudoquasi Type I at $z^{*}$ with

$$
\left(\frac{\rho_{1}^{1}}{\alpha^{1}\left(x^{*}, z^{*}\right)}+\frac{\sum_{\beta=1}^{r} \rho_{\beta}^{2}}{\alpha^{2}\left(x^{*}, z^{*}\right)}\right) \geq 0,
$$

and that $\nabla g_{j}\left(x^{*}\right), j \in J\left(x^{*}\right)$, are linearly independent. Then, $z^{*}=x^{*}$; that is, $z^{*}$ is an optimal solution of (NP).

Proof Suppose to the contrary that $z^{*} \neq x^{*}$ to exhibit a contradiction. Since $x^{*}$ and $\left(z^{*}, u^{*}, \mu^{*}, s^{*}, t^{*}, \tilde{y}^{*}, p^{*}\right)$ are the optimal solutions of (NP) and (GD), respectively, 
and since $\nabla g_{j}\left(x^{*}\right), j \in J\left(x^{*}\right)$ are linearly independent, therefore from the strong duality (Theorem 3.2 ), we obtain

$$
\begin{aligned}
& \sup _{y^{*} \in Y} f\left(x^{*}, y^{*}\right)+\left(x^{* T} B x^{*}\right)^{1 / 2} \\
& =\sum_{i=1}^{s^{*}} t_{i}^{*}\left\{f\left(z^{*}, \bar{y}_{i}^{*}\right)+h\left(z^{*}, \bar{y}_{i}^{*}, p^{*}\right)-p^{* T} \nabla_{p} h\left(z^{*}, \bar{y}_{i}^{*}, p^{*}\right)\right\} \\
& \quad+z^{* T} B u^{*}+\sum_{j \in J_{\circ}}\left\{\mu_{j}^{*} g_{j}\left(z^{*}\right)+\mu_{j}^{*} k_{j}\left(z^{*}, p^{*}\right)-p^{* T} \nabla_{p}\left(\mu_{j}^{*} k_{j}\left(z^{*}, p^{*}\right)\right)\right\} .
\end{aligned}
$$

Thus, we have

$$
\begin{aligned}
& f\left(x^{*}, \bar{y}_{i}^{*}\right)+\left(x^{* T} B x^{*}\right)^{1 / 2} \\
& \leq \sum_{i=1}^{s^{*}} t_{i}^{*}\left\{f\left(z^{*}, \bar{y}_{i}^{*}\right)+h\left(z^{*}, \bar{y}_{i}^{*}, p^{*}\right)-p^{* T} \nabla_{p} h\left(z^{*}, \bar{y}_{i}^{*}, p^{*}\right)\right\} \\
& \quad+z^{* T} B u^{*}+\sum_{j \in J_{\circ}}\left\{\mu_{j}^{*} g_{j}\left(z^{*}\right)+\mu_{j}^{*} k_{j}\left(z^{*}, p^{*}\right)-p^{* T} \nabla_{p}\left(\mu_{j}^{*} k_{j}\left(z^{*}, p^{*}\right)\right)\right\},
\end{aligned}
$$

for all $\bar{y}_{i}^{*} \in Y\left(x^{*}\right), i=1,2, \ldots, s^{*}$.

Now, proceeding as in Theorem 3.1, we get

$$
\begin{aligned}
& \sum_{i=1}^{s^{*}} t_{i}^{*} f\left(x^{*}, \bar{y}_{i}^{*}\right)+x^{* T} B u^{*}+\sum_{j \in J_{\circ}} \mu_{j}^{*} g_{j}\left(x^{*}\right) \\
& <\sum_{i=1}^{s^{*}} t_{i}^{*}\left\{f\left(z^{*}, \bar{y}_{i}^{*}\right)+h\left(z^{*}, \bar{y}_{i}^{*}, p^{*}\right)-p^{* T} \nabla_{p} h\left(z^{*}, \bar{y}_{i}^{*}, p^{*}\right)\right\}+z^{* T} B u^{*} \\
& \quad+\sum_{j \in J_{\circ}}\left\{\mu_{j}^{*} g_{j}\left(z^{*}\right)+\mu_{j}^{*} k_{j}\left(z^{*}, p^{*}\right)-p^{* T} \nabla_{p}\left(\mu_{j}^{*} k_{j}\left(z^{*}, p^{*}\right)\right)\right\} .
\end{aligned}
$$

From (2), we have

$$
-\sum_{j \in J_{\beta}}\left\{\mu_{j}^{*} g_{j}\left(z^{*}\right)+\mu_{j}^{*} k_{j}\left(z^{*}, p^{*}\right)-p^{* T} \nabla_{p}\left(\mu_{j}^{*} k_{j}\left(z^{*}, p^{*}\right)\right)\right\} \leq 0, \quad \beta=1,2, \ldots, r,
$$

which by the second part of higher-order $(F, \alpha, \rho, d)$-strictlypseudoquasi Type I assumption on

$$
\left[\sum_{i=1}^{s^{*}} t_{i}^{*} f\left(\cdot, \bar{y}_{i}^{*}\right)+(\cdot)^{T} B u^{*}+\sum_{j \in J_{\circ}} \mu_{j}^{*} g_{j}(\cdot), \sum_{j \in J_{\beta}} \mu_{j}^{*} g_{j}(\cdot), \beta=1,2, \ldots, r\right]
$$


at $z^{*}$ gives

$$
F\left(x^{*}, z^{*} ; \alpha^{2}\left(x^{*}, z^{*}\right) \sum_{j \in J_{\beta}} \nabla_{p}\left(\mu_{j}^{*} k_{j}\left(z^{*}, p^{*}\right)\right)\right) \leq-\rho_{\beta}^{2} d^{2}\left(x^{*}, z^{*}\right), \quad \beta=1,2, \ldots, r .
$$

As $\alpha^{2}\left(x^{*}, z^{*}\right)>0$ and as $F$ is sublinear, it follows that

$$
F\left(x^{*}, z^{*} ; \sum_{j \in J_{\beta}} \nabla_{p}\left(\mu_{j}^{*} k_{j}\left(z^{*}, p^{*}\right)\right)\right) \leq-\frac{\rho_{\beta}^{2}}{\alpha^{2}\left(x^{*}, z^{*}\right)} d^{2}\left(x^{*}, z^{*}\right), \quad \beta=1,2, \ldots, r .
$$

From the first dual constraint, (14) and the sublinearity of $F$, we have

$$
\begin{aligned}
& F\left(x^{*}, z^{*} ; \sum_{i=1}^{s^{*}} t_{i}^{*} \nabla_{p} h\left(z^{*}, \bar{y}_{i}^{*}, p^{*}\right)+B u^{*}+\sum_{j \in J_{\circ}} \nabla_{p}\left(\mu_{j}^{*} k_{j}\left(z^{*}, p^{*}\right)\right)\right) \\
& \quad \geq \frac{\sum_{\beta=1}^{r} \rho_{\beta}^{2}}{\alpha^{2}\left(x^{*}, z^{*}\right)} d^{2}\left(x^{*}, z^{*}\right) .
\end{aligned}
$$

In view of

$$
\left(\frac{\rho_{1}^{1}}{\alpha^{1}\left(x^{*}, z^{*}\right)}+\frac{\sum_{\beta=1}^{r} \rho_{\beta}^{2}}{\alpha^{2}\left(x^{*}, z^{*}\right)}\right) \geq 0,
$$

$\alpha^{1}\left(x^{*}, z^{*}\right)>0$ and the sublinearity of $F$, the above inequality becomes

$$
\begin{aligned}
& F\left(x^{*}, z^{*} ; \alpha^{1}\left(x^{*}, z^{*}\right)\left\{\sum_{i=1}^{s^{*}} t_{i}^{*} \nabla_{p} h\left(z^{*}, \bar{y}_{i}^{*}, p^{*}\right)+B u^{*}+\sum_{j \in J_{\circ}} \nabla_{p}\left(\mu_{j}^{*} k_{j}\left(z^{*}, p^{*}\right)\right)\right\}\right) \\
& \quad \geq-\rho_{1}^{1} d^{2}\left(x^{*}, z^{*}\right) .
\end{aligned}
$$

On using the first part of the said assumption imposed on

$$
\left[\sum_{i=1}^{s^{*}} t_{i}^{*} f\left(\cdot, \bar{y}_{i}^{*}\right)+(\cdot)^{T} B u^{*}+\sum_{j \in J_{\circ}} \mu_{j}^{*} g_{j}(\cdot), \sum_{j \in J_{\beta}} \mu_{j}^{*} g_{j}(\cdot), \beta=1,2, \ldots, r\right]
$$

at $z^{*}$, it follows that

$$
\begin{aligned}
& \sum_{i=1}^{s^{*}} t_{i}^{*} f\left(x^{*}, \bar{y}_{i}^{*}\right)+x^{* T} B u^{*}+\sum_{j \in J_{\circ}} \mu_{j}^{*} g_{j}\left(x^{*}\right) \\
& \quad>\sum_{i=1}^{s^{*}} t_{i}^{*}\left[f\left(z^{*}, \bar{y}_{i}^{*}\right)+h\left(z^{*}, \bar{y}_{i}^{*}, p^{*}\right)-p^{* T} \nabla_{p} h\left(z^{*}, \bar{y}_{i}^{*}, p^{*}\right)\right] \\
& \quad+z^{* T} B u^{*}+\sum_{j \in J_{\circ}}\left[\mu_{j}^{*} g_{j}\left(z^{*}\right)+\mu_{j}^{*} k_{j}\left(z^{*}, p^{*}\right)-p^{* T} \nabla_{p}\left(\mu_{j}^{*} k_{j}\left(z^{*}, p^{*}\right)\right)\right],
\end{aligned}
$$

which is a contradiction to (13). Hence, $z^{*}=x^{*}$. 


\section{Remark 3.2}

(i) If we take $h\left(z, \bar{y}_{i}, p\right)=p^{T} \nabla f\left(z, \bar{y}_{i}\right)+\frac{1}{2} p^{T} \nabla^{2} f\left(z, \bar{y}_{i}\right) p, i=1,2, \ldots, s$, and $k_{j}(z, p)=p^{T} \nabla g_{j}(z)+\frac{1}{2} p^{T} \nabla^{2} g_{j}(z) p, j \in J$ in Theorems 3.1-3.3, then we get Theorems 4.1-4.3 discussed by Ahmad, Husain and Sharma (Ref. [24]). If, in addition, $\alpha^{1}(x, \bar{x})=1=\alpha^{2}(x, \bar{x}), \rho_{1}^{1}=0=\rho_{\beta}^{2}, \beta=1,2, \ldots, r$ and $F(x, z ; a)=$ $\eta^{T}(x, z) a$ for a certain mapping $\eta: S \times X \rightarrow R^{n}$, we obtain Theorems 3.1-3.3 in (Ref. [18]).

(ii) If we put $h\left(z, \bar{y}_{i}, p\right)=p^{T} \nabla f\left(z, \bar{y}_{i}\right)+\frac{1}{2} p^{T} \nabla^{2} f\left(z, \bar{y}_{i}\right) p, \quad i=1,2, \ldots, s$, $k_{j}(z, p)=p^{T} \nabla g_{j}(z)+\frac{1}{2} p^{T} \nabla^{2} g_{j}(z) p, j \in J$, and $J_{\beta}=\phi, \beta=1,2, \ldots, r$ in Theorems 3.1-3.3, then we obtain Theorems 3.1-3.3 in Ref. [24].

\section{Further Development}

The results appeared in this paper can be further generalized to the following related classes of nondifferentiable minimax programming problems:

$$
\begin{aligned}
& \text { (P1) } \quad \text { Min } \sup _{y \in Y} \frac{\phi(x, y)+\left(x^{T} B x\right)^{1 / 2}}{\psi(x, y)-\left(x^{T} D x\right)^{1 / 2},} \\
& \text { s.t. } \quad g(x) \leq 0, \quad x \in R^{n}, \\
& \text { (P2) } \quad \text { Min } \sup _{v \in W} \frac{\operatorname{Re}\left[\phi(\xi, \nu)+\left(z^{H} B z\right)^{1 / 2}\right]}{\operatorname{Re}\left[\psi(\xi, \nu)-\left(z^{H} D z\right)^{1 / 2}\right]}, \\
& \text { s.t. } \quad-g(\xi) \in S^{\circ}, \quad \xi \in C^{2 n},
\end{aligned}
$$

where $\xi=(z, \bar{z}), v=(\omega, \bar{\omega})$ for $z \in C^{n}, \omega \in C^{l} . \phi(\cdot, \cdot): C^{2 n} \times C^{2 l} \rightarrow C$ and $\psi(\cdot, \cdot)$ : $C^{2 n} \times C^{2 l} \rightarrow C$ are analytic with respect to $\xi, W$ is a specified compact subset in $C^{2 l}, S^{\circ}$ is a polyhedral cone in $C^{m}$ and $g: C^{2 n} \rightarrow C^{m}$ is analytic. Also $B, D \in C^{n \times n}$ are positive semidefinite Hermitian matrices.

\section{References}

1. Schmitendorf, W.E.: Necessary conditions and sufficient conditions for static minimax problems. J. Math. Anal. Appl. 57, 683-693 (1977)

2. Tanimoto, S.: Duality for a class of nondifferentiable mathematical programming problems. J. Math. Anal. Appl. 79, 283-294 (1981)

3. Yadav, S.R., Mukherjee, R.N.: Duality for fractional minimax programming problems. J. Aust. Math. Soc. B 31, 484-492 (1990)

4. Chandra, S., Kumar, V.: Duality in fractional minimax programming. J. Aust. Math. Soc. A 58, 376386 (1995)

5. Liu, J.C., Wu, C.S.: On minimax fractional optimality conditions with $(F, \rho)$-convexity. J. Math. Anal. Appl. 219, 36-51 (1998)

6. Lai, H.C., Liu, J.C., Tanaka, K.: Necessary and sufficient conditions for minimax fractional programming. J. Math. Anal. Appl. 230, 311-328 (1999)

7. Lai, H.C., Lee, J.C.: On duality theorems for a nondifferentiable minimax fractional programming. J. Comput. Appl. Math. 146, 115-126 (2002) 
8. Ahmad, I.: Optimality conditions and duality in fractional minimax programming involving generalized $\rho$-invexity. Int. J. Manag. Syst. 19, 165-180 (2003)

9. Liang, Z.A., Shi, Z.W.: Optimality conditions and duality for a minimax fractional programming with generalized convexity. J. Math. Anal. Appl. 277, 474-488 (2003)

10. Jayswal, A.: Non-differentiable minimax fractional programming with generalized $\alpha$-univexity. J. Comput. Appl. Math. 214, 121-135 (2008)

11. Husain, I., Hanson, M.A., Jabeen, Z.: On nondifferentiable fractional minimax programming. Eur. J. Oper. Res. 160, 202-217 (2005)

12. Yang, X.M., Hou, S.H.: On minimax fractional optimality and duality with generalized convexity. J. Glob. Optim. 31, 235-252 (2005)

13. Ahmad, I., Husain, Z.: Optimality conditions and duality in nondifferentiable minimax fractional programming with generalized convexity. J. Optim. Theory Appl. 129, 255-275 (2006)

14. Ahmad, I., Husain, Z.: Duality in nondifferentiable minimax fractional programming with generalized convexity. Appl. Math. Comput. 176, 545-551 (2006)

15. Yuan, D.H., Liu, X.L., Chinchuluun, A., Pardalos, P.M.: Nondifferentiable minimax fractional programming problems with $(C, \alpha, \rho, d)$-convexity. J. Optim. Theory Appl. 129, 185-199 (2006)

16. Liu, J.C.: Second-order duality for minimax programming. Utilitas Math. 56, 53-63 (1999)

17. Du, D.Z., Pardalos, P.M.: Minimax and Applications. Kluwer Academic, Amsterdam (1995)

18. Mishra, S.K., Rueda, N.G.: Second-order duality for nondifferentiable minimax programming involving generalized Type I functions. J. Optim. Theory Appl. 130, 479-488 (2006)

19. Liang, Z.A., Huang, H.X., Pardalos, P.M.: Optimality conditions and duality for a class of nonlinear fractional programming problems. J. Optim. Theory Appl. 110, 611-619 (2001)

20. Liang, Z.A., Huang, H.X., Pardalos, P.M.: Efficiency conditions and duality for a class of multiobjective fractional programming problems. J. Glob. Optim. 27, 447-471 (2003)

21. Ahmad, I., Husain, Z.: Second-order $(F, \alpha, \rho, d)$-convexity and duality in multiobjective programming. Inf. Sci. 176, 3094-3103 (2006)

22. Hachimi, M., Aghezzaf, B.: Second-order duality in multiobjective programming involving generalized Type I functions. Numer. Funct. Anal. Optim. 25, 725-736 (2004)

23. Ahmad, I., Husain, Z., Sharma, S.: Higher-order duality in nondifferentiable multiobjective programming. Numer. Funct. Anal. Optim. 28, 989-1002 (2007)

24. Ahmad, I., Husain, Z., Sharma, S.: Second-order duality in nondifferentiable minmax programming involving Type I functions. J. Comput. Appl. Math. 215, 91-102 (2008) 